\title{
Osmolality controls the expression of cathelicidin antimicrobial peptide
}

\section{in human macrophages}

1 Youxian $\mathrm{Li}^{1, *}$ and Ingvild B. Johnsen ${ }^{1, *}$

$2{ }^{1}$ Department of Clinical and Molecular Medicine, Faculty of Medicine and Health Sciences,

3 Norwegian University of Science and Technology, Trondheim, 7491, Norway

$4 *$ Correspondence:

5 Youxian Li youxian.li@ntnu.no

6 Ingvild B. Johnsen ingvild.johnsen@ntnu.no 


\section{Abstract}

17 An imbalance between extracellular and intracellular fluid osmolality causes osmotic stress and affects

18 cellular homeostasis. Recent research suggests that osmotic stress is also associated with various innate

19 and adaptive immune responses. Here we present the surprising finding that osmolality tightly controls

20 the expression of cathelicidin antimicrobial peptide (CAMP) in human macrophages. CAMP

21 expression is strongly upregulated under hyperosmotic conditions and downregulated under

22 hypoosmotic conditions. We also provide evidence that this osmolality-mediated antimicrobial

23 response is dependent on nuclear factor of activated T-cells 5 (NFAT5) and mitogen-activated protein

24 kinase (MAPK) p38. Finally, Toll-like receptor (TLR) activation inhibits osmolality-mediated

25 expression of CAMP in human macrophages, suggesting that this osmolality-dependent regulation of

26 CAMP is more relevant under homeostatic conditions, rather than during acute infections. This study

27 expands our knowledge of the regulation of human antimicrobial peptides and highlights osmolality as

28 an important and independent factor shaping host innate immune homeostasis. 


\section{$37 \quad 1 \quad$ Introduction}

38 Osmolality describes the number of solute molecules per solution weight. In biological systems, the

39 semipermeable membranes separate intracellular and extracellular fluid with distinct compositions.

40 Hyperosmotic stress can have detrimental effects on cell homeostasis, causing water efflux and cell

41 shrinkage, oxidative stress, DNA damage, cell cycle delay and cell deaths (Burg, Ferraris et al. 2007,

42 Kuper, Beck et al. 2007, Brocker, Thompson et al. 2012). Mammalian cells have developed various

43 osmoadaptive responses to compensate for the adverse effects due to solute concentration asymmetry.

44 For example, under hyperosmotic conditions the transcription factor nuclear factor of activated T cells-

455 (NFAT5) is activated to induce the expression of genes responsible for the synthesis or transport of

46 uncharged small organic osmolytes such as sorbitol, betaine, and myo-inositol. These organic

47 osmolytes can be accumulated to a high level to equalize intracellular and extracellular osmolality

48 without perturbing macromolecular structure and function (Burg, Kwon et al. 1997).

49 The issue of hyperosmotic stress responses is well characterized in renal inner medulla, where cells are

50 routinely exposed to high and varied levels of sodium and urea due to the operation of urinary

51 concentrating mechanisms (Burg, Kwon et al. 1997, López-Rodríguez, Antos et al. 2004, Burg, Ferraris

52 et al. 2007). However, recent studies provide evidence that osmolality is associated with various

53 immune responses. It has been demonstrated that high salt promotes the induction of pathogenic Th17

54 cells (Kleinewietfeld, Manzel et al. 2013, Wu, Yosef et al. 2013), and dampens the immune suppressive

55 functions of Treg cells (Hernandez, Kitz et al. 2015). In macrophages, hyperosmotic stress (or high

56 sodium in particular) has been shown to promote the expression of pro-inflammatory genes and the

57 production of nitric oxide (NO) upon LPS stimulation (Jantsch, Schatz et al. 2015, Zhang, Zheng et al.

58 2015, Tubbs, Liu et al. 2017), activate NLRP3 and NLRC4 inflammasomes (Ip and Medzhitov 2015),

59 enhance type I interferon signaling (Zhang, Du et al. 2018) and reduce IL-4- and IL-13-mediated

60 alternative (M2) activation (Binger, Gebhardt et al. 2015). 
61 Antimicrobial peptides (AMPs) such as defensins and cathelicidins comprise a major component of

62 innate immunity in mammals (Hilchie, Wuerth et al. 2013, Hancock, Haney et al. 2016). Human

63 cathelicidin antimicrobial peptide (CAMP), also known as hCAP-18/LL-37/FALL-39, is the only

64 known human cathelicidin. Human CAMP gene encodes the full-length 18k Da precursor, hCAP-18,

65 which contains a conserved cathelin domain at the N-terminal and the functional antimicrobial domain

66 at the C-terminal (Vandamme, Landuyt et al. 2012). The mature form of CAMP (LL-37) is released

67 from hCAP-18 precursor through a proteinase 3-mediated cleavage process (Sørensen, Follin et al.

68 2001). CAMP has been shown to possess direct antimicrobial activities as well as various

69 immunomodulatory properties (Yang, Chen et al. 2000, Scott, Davidson et al. 2002, Koczulla, Von

70 Degenfeld et al. 2003, Vandamme, Landuyt et al. 2012). In humans CAMP expression is directly

71 regulated by vitamin D signaling (Wang, Nestel et al. 2004, Gombart, Borregaard et al. 2005), although

72 vitamin D-independent regulatory mechanisms have also been proposed (Park, Elias et al. 2011, Guo,

73 Rosoha et al. 2013).

74 In this short report, we show that CAMP expression is tightly regulated by osmolality in human

75 macrophages and reveal that NFAT5 and p38 kinase are the key components of the signaling pathway.

76 We also demonstrate that canonical Toll-like receptor (TLR) activation interferes with this osmolality-

77 driven antimicrobial response. Our study opens up a new understanding of innate immune signaling,

78 demonstrating for the first time that a change in environmental osmolality alone is sufficient to elicit

79 an antimicrobial response in human macrophages.

\section{$80 \quad$ Results}

\section{Osmolality controls CAMP expression in human macrophages}

82 We cultured human monocyte-derived macrophages (MDMs) under hyperosmotic (adjusted by $\mathrm{NaCl}$ 83 or sucrose) or hypoosmotic conditions (adjusted by $\mathrm{H}_{2} \mathrm{O}$ ) to evaluate the effect of osmolality on CAMP 
expression. Active vitamin $\mathrm{D}\left(1,25(\mathrm{OH})_{2} \mathrm{D}_{3}\right)$, a known inducer of CAMP expression in human

avoid any effect mediated by serum vitamin D all cells were cultured under serum-free conditions in

87 this study. As shown in Figure 1A-C, hyperosmolality upregulated CAMP mRNA and protein

88 expression in a dose-dependent manner. Notably, a modest increase in osmolality by $50 \mathrm{mOsm}$ or $100 \mathrm{mOsm}$ was sufficient to induce higher levels of CAMP comparing to $1,25(\mathrm{OH})_{2} \mathrm{D}_{3}$ treatment. $\mathrm{NaCl}$ and sucrose showed similar inductive effects, indicating that this osmolality-mediated CAMP response

91 is independent of the types of osmolytes. Interestingly, CAMP expression was reduced in macrophages with hypoosmotic treatment, suggesting that osmolality contributes to basal CAMP expression even at

93 physiologically isosmotic conditions. Taken together these data indicate that osmolality effectively 94 controls CAMP expression in human macrophages.

Osmolality-mediated CAMP expression in human macrophages are regulated by NFAT5 and p38

We went on to investigate the molecular mechanisms underlying osmolality-mediated CAMP response. As NFAT5 is a central component of osmoadaptive signaling, we used NFAT5 siRNA (siNFAT5) to knock down NFAT5 gene expression before we manipulated medium osmolality. siNFAT5 effectively suppressed NFAT5 gene expression before hyperosmotic treatment (Figure 2A). Prior knockdown of NFAT5 expression strongly repressed basal and hyperosmolality-induced CAMP

102 expression (Figure 2B, C), indicating that CAMP expression is controlled by osmolality via a NFAT5-

103 dependent mechanism. Interestingly, hyperosmotic treatment did not enhance NFAT5 expression in 104 control siRNA-treated cells, but promoted NFAT5 expression in siNFAT5-treated cells 105 (Supplementary Figure 1A, B). This suggests that the constitutive expression of NFAT5 in human 106 macrophages is normally sufficient, but a feedback loop exists so that when cells with insufficient

107 NFAT5 levels sense the need to elicit osmoadaptive responses, NFAT5 expression can be levelled up. 
108 The molecular mechanisms underlying NFAT5 activation is not well understood, however it has been

109 shown that $\mathrm{p} 38$, a subgroup of mitogen-activated protein kinases (MAPKs), is required for osmolality-

110 dependent activation of NFAT5 (Ko, Lam et al. 2002, Zhou 2015, Zhou 2016). Therefore, we examined

111 if p38 contributes to osmolality-meidated CAMP expression. We used two selective p38 kinase

112 inhibitors, SB202190 and SB203580 (Wilson, McCaffrey et al. 1997, Young, McLaughlin et al. 1997),

113 to block p38 kinase activity. SB202190 and SB203580 pretreatment strongly reduced basal and

114 hyperosmolality-induced CAMP expression both at the mRNA level (Figure 2D, E) and at the protein

115 level (Figure 2F, G), indicating that p38 kinase activity is also important for the signaling of osmolality-

116 mediated CAMP expression.

117 Toll-like receptor (TLR) activation inhibits osmolality-mediated CAMP expression in human

118 macrophages

119 High salt has been reported to potentiate the expression of various cytokines and pro-inflammatory

120 genes when macrophages are activated by TLR ligands such as LPS (Jantsch, Schatz et al. 2015, Zhang,

121 Zheng et al. 2015, Tubbs, Liu et al. 2017). We next asked if TLR stimulation affects osmolality-

122 mediated CAMP response in human macrophages. We treated macrophages with two TLR ligands:

123 Pam3CSK4 (a TLR1/2 ligand) and LPS (a TLR4 ligand), and cultured the cells under isosmotic or

124 hyperosmotic conditions. Interestingly, both Pam3CSK4 and LPS treatment strongly reduced basal and

125 hyperosmolality-induced CAMP expression (Figure 3A-C). Pam3CSK4 and LPS did not downregulate

126 NFAT5 gene expression in macrophages (Figure 3D), suggesting that the suppressive effect of TLR

127 agonists may be modulated via post-transcriptional regulation of NFAT5 (e.g., inhibition of NFAT5

128 protein activation), or via a NFAT5-independent mechanism. An earlier report (Dhawan, Wei et al.

129 2015) and our recent study ( $\mathrm{Li}, \varnothing$ sterhus et al. 2018) have shown that the transcription factor $\mathrm{C} / \mathrm{EBP} \alpha$

130 plays a critical role in mediating CAMP expression in human airway epithelial cells and macrophages.

131 Therefore, we examined the expression of C/EBP $\alpha$ in macrophages upon Pam3CSK4 or LPS treatment. 
132 As shown in Figure 3E, C/EBP $\alpha$ expression was repressed by LPS or Pam3CSK4 stimulation,

133 corresponding to downregulated CAMP expression (Figure 3A-C). Taken together our data suggest

134 that TLR activation suppresses osmolality-mediated CAMP expression possibly via downregulation of $135 \mathrm{C} / \mathrm{EBP} \alpha$.

\section{Discussion}

137 The molecular mechanisms underlying macrophage activation and defense gene expression have been 138 extensively studied over the last two decades. Although the canonical structural recognition of 139 pathogen-associated molecular patterns (PAMPs) or danger-associated molecular patterns (DAMPs)

140 and the downstream signaling pathways are well-characterized, accumulating evidence suggests that

141 macrophages can also sense and respond to environmental changes, such as nutrient availability and 142 oxygen levels (Strehl, Fangradt et al. 2014, O’Neill and Pearce 2016, Langston, Shibata et al. 2017).

143 The association between osmolality and macrophage functions is only beginning to be appreciated 144 (Binger, Gebhardt et al. 2015, Ip and Medzhitov 2015, Jantsch, Schatz et al. 2015, Zhang, Zheng et al. 145 2015, Tubbs, Liu et al. 2017, Zhang, Du et al. 2018). These studies suggest that hyperosmotic stress 146 (or high $\mathrm{NaCl}$ specifically) tends to polarize macrophages towards a more pro-inflammatory 147 phenotype. Our report however indicates that osmolality is more than a factor potentiating

148 inflammation. We provide clear evidence that human macrophages can effectively monitor osmolality 149 changes in the environment to elicit (or repress) an important antimicrobial response, in the absence of 150 other inflammatory stimuli.

151 The following observations make this newly identified regulatory mechanism of CAMP particularly 152 intriguing. First, osmolality can both positively and negatively affect CAMP expression: 153 hyperosmolality upregulates CAMP expression whilst hypoosmolality downregulates CAMP 154 expression, suggesting that this is a homeostatic regulatory mechanism adapting macrophage CAMP 155 expression levels to osmolality changes in the environment. Secondly, CAMP expression is very 
sensitive to changes in osmolality: a modest increase by 50 or $100 \mathrm{mOsm}$ induces higher expression of

157 CAMP comparing to active vitamin D treatment. Thirdly, hyperosmolality induces CAMP expression

158 via a shared mechanism regardless of the types of osmolytes, as both the ionic and non-ionic osmolytes

159 ( $\mathrm{NaCl}$ and sucrose) potently induce CAMP expression via the same NFAT5- and p38-dependent

160 signaling pathway. Finally, hyperosmolality alone is sufficient to drive CAMP expression without the

161 need of additional stimulation (e.g., LPS treatment). In fact CAMP expression is strongly reduced when

162 macrophages are primed by Pam3CSK4 or LPS, emphasizing that this osmolality-mediated CAMP

163 response is a kind of homeostatic regulation (and possibly a preventive defense mechanism), rather

164 than an acute response to infections. This observation supports what we have proposed earlier ( $\mathrm{Li}$,

165 Østerhus et al. 2018), that human macrophages express CAMP under homeostatic conditions, but shift

166 away from CAMP production to prioritize production of other effector molecules such as cytokines

167 and chemokines when encountering a serious pathogenic threat.

168 The physiological implications of this osmolality-dependent regulation of CAMP remain to be

169 elucidated. It will also be interesting to investigate if this regulatory mechanism of AMPs is present in

170 species other than humans. Given the prominent antimicrobial property of CAMP, it is tempting to

171 speculate that this regulatory mechanism may be particularly relevant for local defense in tissues with

172 significant fluctuations in osmolality, such as the gut and the urinary system. Our study will likely

173 provide useful insights into the maintenance and breakdown of immune homeostasis in these tissues.

174 In addition, this newly identified osmolality-dependent regulation offers a potentially simple and

175 practical approach to manipulate CAMP levels in vivo, either for investigative purpose to reveal CAMP

176 functions that are truly physiologically relevant, or for potential therapeutic purposes to boost natural 
Materials and methods

\section{Reagents}

182 Active vitamin $\mathrm{D}\left(1,25(\mathrm{OH})_{2} \mathrm{D}_{3}\right)$ was purchased from Tocris Bioscience and used at a working

183 concentration of 100nM. Pam3CSK4 was purchased from Invivogen and used at a working

184 concentration of $1 \mu \mathrm{g} / \mathrm{mL}$. LPS was purchased from Sigma Aldrich and used at a working concentration

185 of $1 \mu \mathrm{g} / \mathrm{mL}$. SB202190 and SB203580 were purchased from Sigma Aldrich and used at working

186 concentrations of $10 \mu \mathrm{M}$ and $20 \mu \mathrm{M}$ respectively (30 min pretreatment).

\section{Cell culture and medium osmolality adjustment.}

188 Peripheral blood mononuclear cells (PBMCs) were isolated from fresh buffy coats of healthy donors

189 using gradient centrifugation with Lymphoprep ${ }^{\mathrm{TM}}$ (Axis-Shield). Buffy coats were supplied by the

190 blood bank at St.Olavs Hospital in Trondheim, Norway, and their use in research has been approved

191 by the Regional Committee for Medical and Health Research Ethics (REK), and by the donors

192 themselves. Cells were washed with PBS and seeded in RPMI 1640 medium (supplemented with 0.34

$193 \mathrm{mM}$ L-glutamine and $10 \mu \mathrm{g} / \mathrm{mL}$ gentamicin). After two hours non-adherent cells were removed by

194 washing with RPMI 1640 medium. Monocytes were cultivated in RPMI 1640 medium supplemented

195 with $10 \%$ human serum (heat inactivated, obtained from blood bank of St. Olavs Hospital, Trondheim),

$1960.34 \mathrm{mM}$ L-glutamine, $10 \mu \mathrm{g} / \mathrm{mL}$ gentamicin and $10 \mathrm{ng} / \mathrm{mL}$ M-CSF (Biolegend) for macrophage

197 differentiation. Medium was changed every three days. Macrophages differentiated for 12-16 days

198 were used in this study. On the day of treatment, medium was switched to serum-free RPMI 1640

199 (with or without osmolality adjustment). Medium osmolality was adjusted by sterilized deionized

200 water, $300 \mathrm{mM}$ sucrose (in RPMI) or $150 \mathrm{mM} \mathrm{NaCl}$ (in RPMI). After 24h samples were collected for

201 quantitative real-time PCR or Western blot analysis. 


\section{Quantitative real-time PCR (qRT-PCR)}

204 RNA was isolated with the RNeasy mini kit (Qiagen) following the manufacturer's protocol. cDNA

205 was synthesized from isolated RNA using the qScript kit (Quanta) following the manufacturer's

206 protocol. Quantitative real-time PCR (qRT-PCR) was performed using Perfecta SYBR Green reaction

207 mix (Quanta) and a StepOnePlus instrument (Life Technologies) with the temperature profile $95^{\circ} \mathrm{C}$ for

$20820 \mathrm{~s}, 40$ cycles at $95^{\circ} \mathrm{C}$ for $3 \mathrm{~s}$ and $60^{\circ} \mathrm{C}$ for 30 s. Fold change in gene expression was calculated using

209 the $\Delta \Delta \mathrm{Ct}$-method normalized against GAPDH. Sequences of primers used in this study are as follows:

210 CAMP: forward 5'-TCGGATGCTAACCTCTACCG-3', reverse 5'-GTCTGGGTCCCCATCCAT-

$211 \quad 3^{\prime} ; \quad$ GAPDH: forward 5'-GAAGGTGAAGGTCGGAGTC-3', $\quad$ reverse $\quad 5^{\prime}-$

212 GAAGATGGTGATGGGATTTC-3’' CEBPA: forward 5’-GGAGCTGAGATCCCGACA-3’, reverse

213 5’-TTCTAAGGACAGGCGTGGAG-3'

214 Western Blot

215 Cells were washed once in PBS and lysed in lysis buffer (50mM Tris- $\mathrm{HCl}, 150 \mathrm{mM} \mathrm{NaCl}, 10 \%$

216 Glycerol, 0,5\% Triton X-100 and 2mM EDTA) containing phosphatase and protease inhibitors (100

$217 \mathrm{mM}$ Sodium Fluoride, $1 \mathrm{mM}$ Sodium Orthovanadate, $40 \mathrm{mM}$-Glycerophosphate, $10 \mu \mathrm{g} / \mathrm{mL}$

218 Leupeptin, $1 \mu \mathrm{M}$ Pepstatin $\mathrm{A}$ and $1 \mathrm{mM}$ Phenylmethylsulfonyl fluoride). Protein extracts were

219 separated by NuPAGE® Bis-Tris gels (Thermo Fisher Scientific) and dry blotting was performed using

220 iBlot ${ }^{\circledR}$ Gel Transfer stacks Nitrocellulose Mini kit and iBlot ${ }^{\circledR}$ machine (Invitrogen). Primary human

221 antibodies for hCAP-18 (\#650302) and NFAT5 (\#F-9) were purchased from Biolegend and Santa Cruz

222 respectively. Household $\beta$-actin antibody (A1978) was purchased from Sigma Aldrich and used as a

223 loading control. Secondary antibodies (IRDye® 800CW Goat anti-Mouse, IRDye® 680RD Goat anti-

224 Mouse) were purchased from LI-COR Biosciences. LICOR Odyssey imager was used as the scanning 225 system.

\section{RNA interference}


siRNAs were purchased from Qiagen (AllStars control siRNA) and Ambion (NFAT5) respectively.

Scientific) transfection reagent according to the manufacturer's instructions. Cells were incubated with

siRNA-RNAiMAX complex for 72 hours before further treatment.

\section{Statistics}

232 Results are expressed as mean $+\mathrm{SD}(\mathrm{n}=3)$. A two-sided P-value $<0.05$ as determined by Student t-test

was considered significant. All data are representative of at least three independent experiments with

PBMCs from different donors.

\section{References}

Binger, K. J., M. Gebhardt, M. Heinig, C. Rintisch, A. Schroeder, W. Neuhofer, K. Hilgers, A. Manzel, C.

Schwartz and M. Kleinewietfeld (2015). "High salt reduces the activation of IL-4-and IL-13-stimulated

238

239

240

241 macrophages." The Journal of clinical investigation 125(11): 4223-4238.

Brocker, C., D. C. Thompson and V. Vasiliou (2012). "The role of hyperosmotic stress in inflammation and disease." Biomolecular concepts 3(4): 345-364.

Burg, M. B., J. D. Ferraris and N. I. Dmitrieva (2007). "Cellular response to hyperosmotic stresses." Physiological reviews 87(4): 1441-1474.

Burg, M. B., E. D. Kwon and D. KÜltz (1997). "Regulation of gene expression by hypertonicity." Annual Review of Physiology 59(1): 437-455. the Vitamin D Receptor Cooperate in the Regulation of Cathelicidin in Lung Epithelial Cells." Journal of cellular physiology 230(2): 464-472.

Gombart, A. F., N. Borregaard and H. P. Koeffler (2005). "Human cathelicidin antimicrobial peptide (CAMP) gene is a direct target of the vitamin $D$ receptor and is strongly up-regulated in myeloid cells by 1,25 dihydroxyvitamin D3." The FASEB journal 19(9): 1067-1077.

Guo, C., E. Rosoha, M. B. Lowry, N. Borregaard and A. F. Gombart (2013). "Curcumin induces human cathelicidin antimicrobial peptide gene expression through a vitamin D receptor-independent pathway." The Journal of nutritional biochemistry 24(5): 754-759.

Hancock, R. E., E. F. Haney and E. E. Gill (2016). "The immunology of host defence peptides: beyond antimicrobial activity." Nature Reviews Immunology 16(5): 321.

Hernandez, A. L., A. Kitz, C. Wu, D. E. Lowther, D. M. Rodriguez, N. Vudattu, S. Deng, K. C. Herold, V. K. Kuchroo and M. Kleinewietfeld (2015). "Sodium chloride inhibits the suppressive function of FOXP3+ regulatory T cells." The Journal of clinical investigation 125(11): 4212-4222.

Hilchie, A. L., K. Wuerth and R. E. Hancock (2013). "Immune modulation by multifaceted cationic host defense (antimicrobial) peptides." Nature chemical biology 9(12): 761.

Ip, W. E. and R. Medzhitov (2015). "Macrophages monitor tissue osmolarity and induce inflammatory response through NLRP3 and NLRC4 inflammasome activation." Nature communications 6: 6931. Jantsch, J., V. Schatz, D. Friedrich, A. Schröder, C. Kopp, I. Siegert, A. Maronna, D. Wendelborn, P. Linz and K. J. Binger (2015). "Cutaneous $\mathrm{Na}$ + storage strengthens the antimicrobial barrier function of the skin and boosts macrophage-driven host defense." Cell metabolism 21(3): 493-501. 
Kleinewietfeld, M., A. Manzel, J. Titze, H. Kvakan, N. Yosef, R. A. Linker, D. N. Muller and D. A. Hafler (2013).

"Sodium chloride drives autoimmune disease by the induction of pathogenic T H 17 cells." Nature 496(7446): 518. required for maximal hypertonic activation of the osmotic response element-binding protein/tonicityresponsive enhancer-binding protein (OREBP/TonEBP)." Journal of Biological Chemistry 277(48): 4608546092. and C. Lebherz (2003). "An angiogenic role for the human peptide antibiotic LL-37/hCAP-18." The Journal of clinical investigation 111(11): 1665-1672.

Kuper, C., F.-X. Beck and W. Neuhofer (2007). "Osmoadaptation of Mammalian cells-an orchestrated network of protective genes." Current genomics 8(4): 209-218.

Langston, P. K., M. Shibata and T. Horng (2017). "Metabolism supports macrophage activation." Frontiers in immunology 8: 61.

Li, Y., S. Østerhus and I. B. Johnsen (2018). "Human metapneumovirus infection inhibits cathelicidin antimicrobial peptide expression in human macrophages." Frontiers in Immunology $\mathbf{9}$.

Liu, P. T., S. Stenger, H. Li, L. Wenzel, B. H. Tan, S. R. Krutzik, M. T. Ochoa, J. Schauber, K. Wu and C. Meinken (2006). "Toll-like receptor triggering of a vitamin D-mediated human antimicrobial response." Science 311(5768): 1770-1773.

López-Rodríguez, C., C. L. Antos, J. M. Shelton, J. A. Richardson, F. Lin, T. I. Novobrantseva, R. T. Bronson, P. Igarashi, A. Rao and E. N. Olson (2004). "Loss of NFAT5 results in renal atrophy and lack of tonicity-responsive gene expression." Proceedings of the National Academy of Sciences of the United States of America 101(8): 2392-2397.

O'Neill, L. A. and E. J. Pearce (2016). "Immunometabolism governs dendritic cell and macrophage function." Journal of Experimental Medicine 213(1): 15-23.

Park, K., P. M. Elias, Y. Oda, D. Mackenzie, T. Mauro, W. M. Holleran and Y. Uchida (2011). "Regulation of cathelicidin antimicrobial peptide expression by an endoplasmic reticulum (ER) stress signaling, vitamin D receptor-independent pathway." Journal of Biological Chemistry 286(39): 34121-34130.

Scott, M. G., D. J. Davidson, M. R. Gold, D. Bowdish and R. E. Hancock (2002). "The human antimicrobial peptide LL-37 is a multifunctional modulator of innate immune responses." The Journal of Immunology 169(7): 3883-3891.

Strehl, C., M. Fangradt, U. Fearon, T. Gaber, F. Buttgereit and D. J. Veale (2014). "Hypoxia: how does the monocyte - macrophage system respond to changes in oxygen availability?" Journal of leukocyte biology 95(2): 233-241.

Sørensen, O. E., P. Follin, A. H. Johnsen, J. Calafat, G. S. Tjabringa, P. S. Hiemstra and N. Borregaard (2001). "Human cathelicidin, hCAP-18, is processed to the antimicrobial peptide LL-37 by extracellular cleavage with proteinase 3." Blood 97(12): 3951-3959.

Tubbs, A. L., B. Liu, T. D. Rogers, R. B. Sartor and E. A. Miao (2017). "Dietary salt exacerbates experimental colitis." The Journal of Immunology 199(3): 1051-1059.

Vandamme, D., B. Landuyt, W. Luyten and L. Schoofs (2012). "A comprehensive summary of LL-37, the factotum human cathelicidin peptide." Cellular immunology 280(1): 22-35.

Wang, T.-T., F. P. Nestel, V. Bourdeau, Y. Nagai, Q. Wang, J. Liao, L. Tavera-Mendoza, R. Lin, J. H. Hanrahan and S. Mader (2004). "Cutting edge: 1, 25-dihydroxyvitamin D3 is a direct inducer of antimicrobial peptide gene expression." The Journal of Immunology 173(5): 2909-2912.

Wilson, K. P., P. G. McCaffrey, K. Hsiao, S. Pazhanisamy, V. Galullo, G. W. Bemis, M. J. Fitzgibbon, P. R. Caron, M. A. Murcko and M. S. Su (1997). "The structural basis for the specificity of pyridinylimidazole inhibitors of p38 MAP kinase." Chemistry \& biology 4(6): 423-431.

Wu, C., N. Yosef, T. Thalhamer, C. Zhu, S. Xiao, Y. Kishi, A. Regev and V. K. Kuchroo (2013). "Induction of pathogenic T H 17 cells by inducible salt-sensing kinase SGK1." Nature 496(7446): 513. 
315 Yang, D., Q. Chen, A. P. Schmidt, G. M. Anderson, J. M. Wang, J. Wooters, J. J. Oppenheim and O. Chertov

316 (2000). "LL-37, the neutrophil granule-and epithelial cell-derived cathelicidin, utilizes formyl peptide

317 receptor-like 1 (FPRL1) as a receptor to chemoattract human peripheral blood neutrophils, monocytes, and T

318 cells." Journal of Experimental Medicine 192(7): 1069-1074.

319 Young, P. R., M. M. McLaughlin, S. Kumar, S. Kassis, M. L. Doyle, D. McNulty, T. F. Gallagher, S. Fisher, P. C.

320 McDonnell and S. A. Carr (1997). "Pyridinyl imidazole inhibitors of p38 mitogen-activated protein kinase bind

321 in the ATP site." Journal of Biological Chemistry 272(18): 12116-12121.

322 Yuk, J.-M., D.-M. Shin, H.-M. Lee, C.-S. Yang, H. S. Jin, K.-K. Kim, Z.-W. Lee, S.-H. Lee, J.-M. Kim and E.-K. Jo

323 (2009). "Vitamin D3 induces autophagy in human monocytes/macrophages via cathelicidin." Cell host \&

324 microbe 6(3): 231-243.

325 Zhang, W.-C., L.-J. Du, X.-J. Zheng, X.-Q. Chen, C. Shi, B.-Y. Chen, X.-N. Sun, C. Li, Y.-Y. Zhang and Y. Liu (2018).

326 "Elevated sodium chloride drives type I interferon signaling in macrophages and increases antiviral

327 resistance." Journal of Biological Chemistry 293(3): 1030-1039.

328 Zhang, W.-C., X.-J. Zheng, L.-J. Du, J.-Y. Sun, Z.-X. Shen, C. Shi, S. Sun, Z. Zhang, X.-q. Chen and M. Qin (2015).

329 "High salt primes a specific activation state of macrophages, M (Na)." Cell research 25(8): 893.

330 Zhou, X. (2015). "Regulation of tonicity-dependent activation of NFAT5 by mitogen-activated protein

331 kinases." Abdomen 2.

332 Zhou, X. (2016). "How do kinases contribute to tonicity-dependent regulation of the transcription factor

333 NFAT5?" World journal of nephrology 5(1): 20. 


\section{Data availability}

346 Data supporting the conclusions of this manuscript will be made available by the authors, without

347 undue reservation, to any qualified researcher.

349 Acknowledgements

350 The work was funded by the Research Council of Norway (grant number 230381). We thank Kristin

351 Rian for excellent technical support.

\section{Author contributions statement}

354 Y.L. conducted the experiments. Y.L. and I.B.J. analyzed the results. Y.L. wrote the manuscript. Both

355 authors reviewed and approved the manuscript.

\section{Competing interests}

358 The authors declare that the research was conducted in the absence of any commercial or financial

359 relationships that could be construed as a potential conflict of interest.

\section{$361 \quad$ Ethics Statement}

362 Buffy coats used in this study were supplied by the blood bank at St. Olavs Hospital in Trondheim,

363 Norway, and their use in research has been approved by the Regional Committee for Medical and

364 Health Research Ethics (REK), and by the donors themselves. 


\section{Figure legends}

367 Figure 1: Osmolality controls CAMP expression in human macrophages

368 (A-C) MDMs were cultured in control medium with no osmolality adjustment (Con), hyperosmotic

369 medium (adjusted by $\mathrm{NaCl}$ or sucrose), hypoosmotic medium (adjusted by $\mathrm{H}_{2} \mathrm{O}$ ), or treated with

$370100 \mathrm{nM} 1,25(\mathrm{OH})_{2} \mathrm{D}_{3}(\mathrm{VD})$ for 24 hours. CAMP mRNA (A) and protein expression (hCAP-18) $(\mathrm{B}, \mathrm{C})$

371 was assessed by qRT-PCR and Western Blot. Error bars represent SD for triplicates. **p $<0.01, * * * p$

$372<0.001$ (compared to Con). Data representative of at least three independent experiments from

373 different donors.

374 Figure 2: Osmolality-mediated CAMP expression in human macrophages are regulated by

$375 \quad$ NFAT5 and p38

376 (A) MDMs were transfected with siRNA targeting NFAT5 (siNFAT5) or scrambled control siRNA

377 (siControl) for 72 hours. NFAT5 mRNA expression was assessed by qRT-PCR. (B, C) MDMs were 378 transfected with siRNA targeting NFAT5 (siNFAT5) or scrambled control siRNA (siControl) for 72

379 hours before cultured in control medium with no osmolality adjustment (Con), or medium with

380 additional $100 \mathrm{mOsm} \mathrm{NaCl}$ or sucrose for $24 \mathrm{~h}$. CAMP mRNA expression (B) was assessed by qRT-

381 PCR and CAMP (hCAP-18) protein expression was assessed by Western Blot (C). (D-G) MDMs were

382 pretreated with vehicle, or p38 kinase inhibitor SB202190 (D, F) or SB203580 (E, G) for 30min, then

383 cultured in control medium with no osmolality adjustment (Con), or medium with additional 100mOsm

$384 \mathrm{NaCl}$ or sucrose. CAMP mRNA $(\mathrm{D}, \mathrm{E})$ and protein expression (hCAP-18) $(\mathrm{F}, \mathrm{G})$ was assessed by qRT-

385 PCR and Western Blot. Error bars represent SD for triplicates. ***p $<0.001$ (comparing to

386 corresponding siControl or vehicle samples). \#\#\# <0.001. Data are representative of at least three

387 independent experiments from different donors. 


\section{human macrophages}

390 (A-C) MDMs were cultured in control medium with no osmolality adjustment (Con), or medium with

391 additional $100 \mathrm{mOsm} \mathrm{NaCl}$ or sucrose, concomitant with mock treatment (N.T.) or treatment with

$3921 \mu \mathrm{g} / \mathrm{mL}$ Pam3CSK4 (Pam3) or LPS. CAMP mRNA (A) and protein expression (hCAP-18) (B, C) was

393 assessed by qRT-PCR and Western Blot. (D, E) MDMs were mock-treated (N.T.) or treated with

$3941 \mu \mathrm{g} / \mathrm{mL}$ Pam3CSK4 (Pam3) or LPS, NFAT5 (D) and C/EBP $\alpha$ (E) mRNA expression was assessed by

395 qRT-PCR. Error bars represent SD for triplicates. **p $<0.01$, ***p $<0.001$ (comparing to

396 corresponding N.T. samples); \#\#\#p <0.001. Data are representative of at least three independent

397 experiments from different donors.

reverses the suppressive effect of NFAT5 siRNA in human macrophages.

400 (A, B) MDMs were transfected with siRNA targeting NFAT5 (siNFAT5) or scrambled control siRNA

401 (siControl) for 72 hours before cultured in control medium with no osmolality adjustment (Con), or

402 medium with additional $100 \mathrm{mOsm} \mathrm{NaCl}$ or sucrose for $24 \mathrm{~h}$. NFAT5 mRNA expression (A) was

403 assessed by qRT-PCR and CAMP (hCAP-18) protein expression was assessed by Western Blot (B).

$404 * * * \mathrm{p}<0.001$ (compared to the corresponding siControl sample). \#\#p $<0.01$. \#\#\#p $<0.001$. Data

405 representative of at least three independent experiments from different donors. 
A

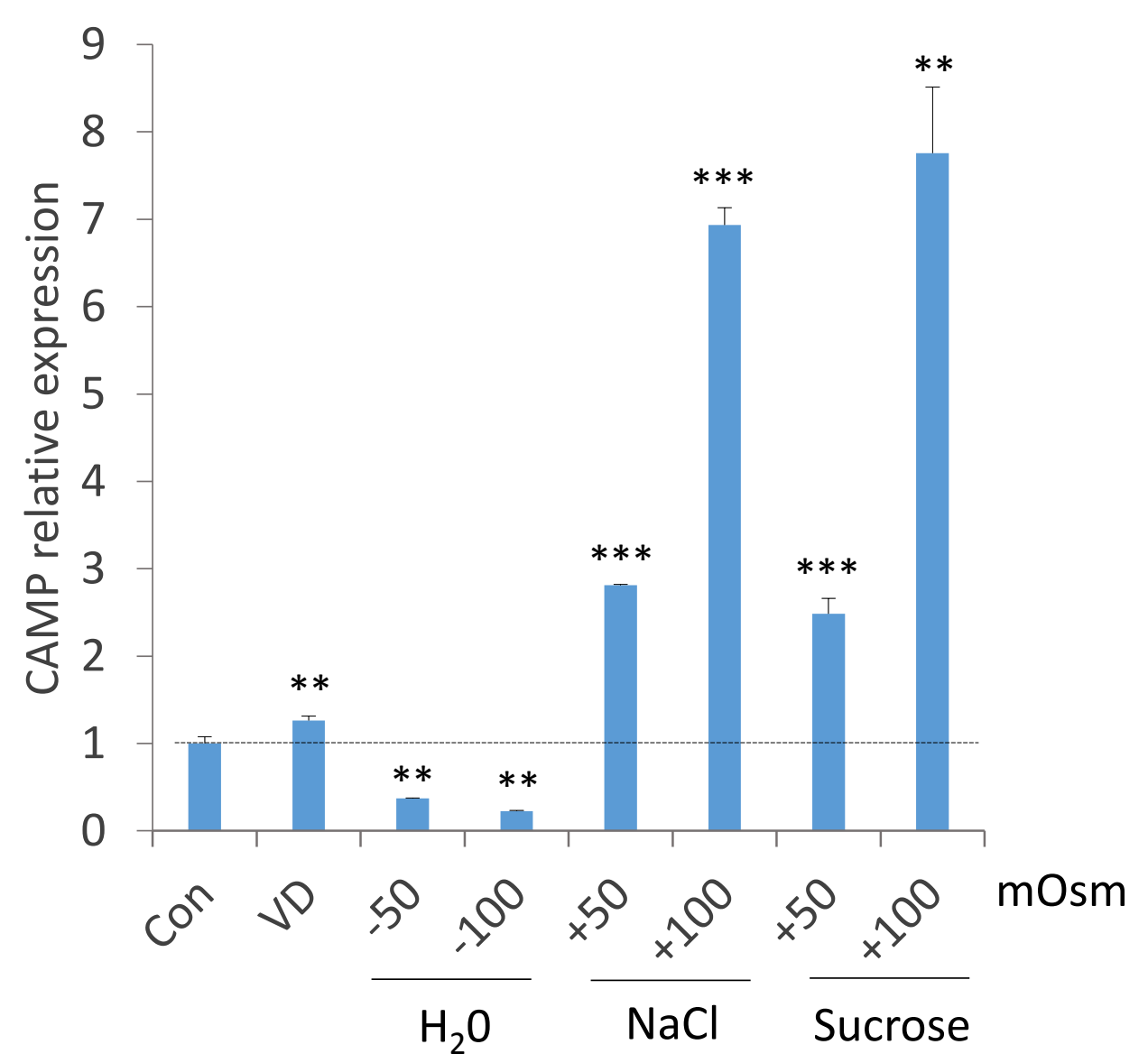

B

\begin{tabular}{|c|c|c|c|c|c|c|c|c|}
\hline \multirow[b]{2}{*}{ mOsm } & \multicolumn{3}{|c|}{$\mathrm{H}_{2} \mathrm{O}$} & \multirow[b]{2}{*}{ Con } & \multicolumn{3}{|c|}{$\mathrm{NaCl}$} & \\
\hline & -150 & -100 & -50 & & +50 & +100 & +150 & VD \\
\hline hCAP-18 & 5 & $\sin$ & $\mathrm{es}$ & & & & & \\
\hline
\end{tabular}

C

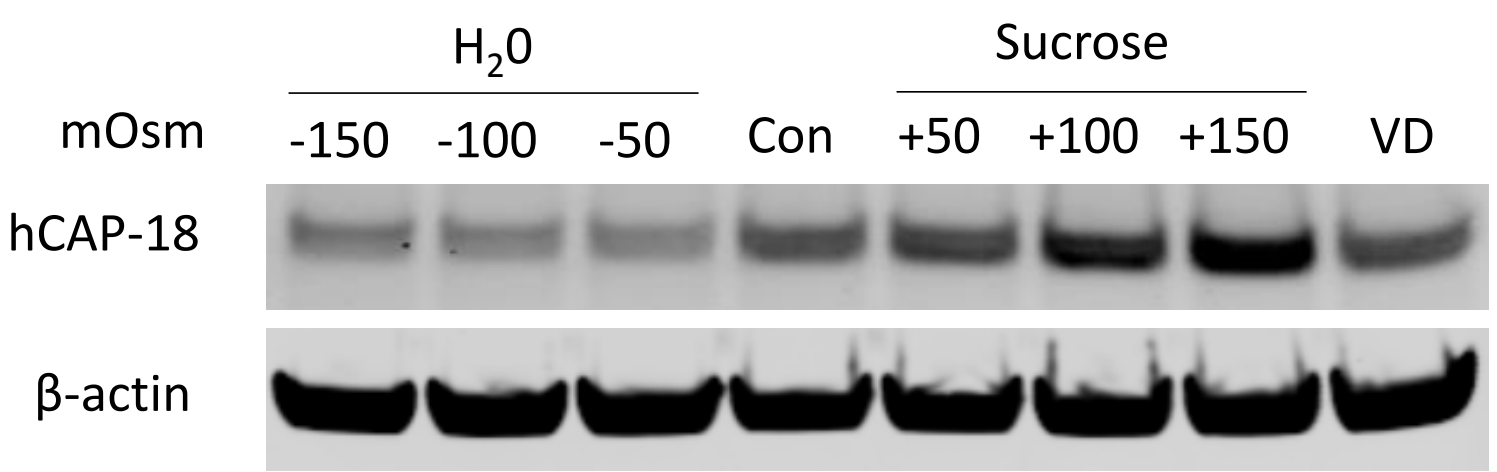

Figure 1 


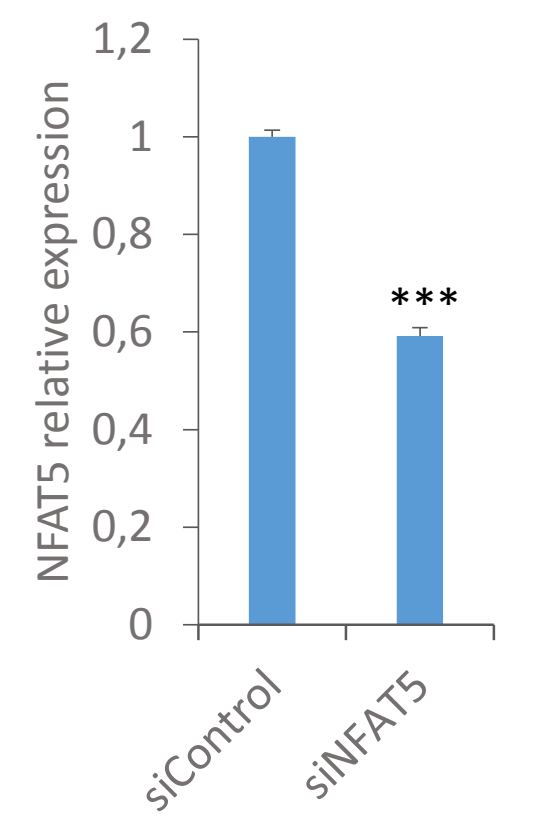

D

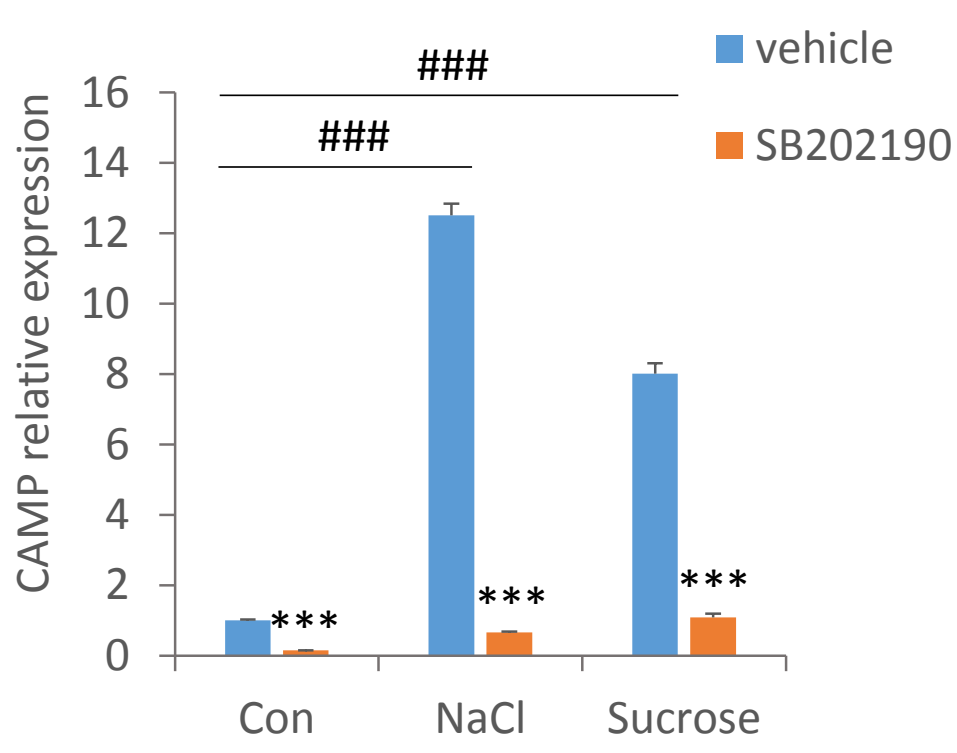

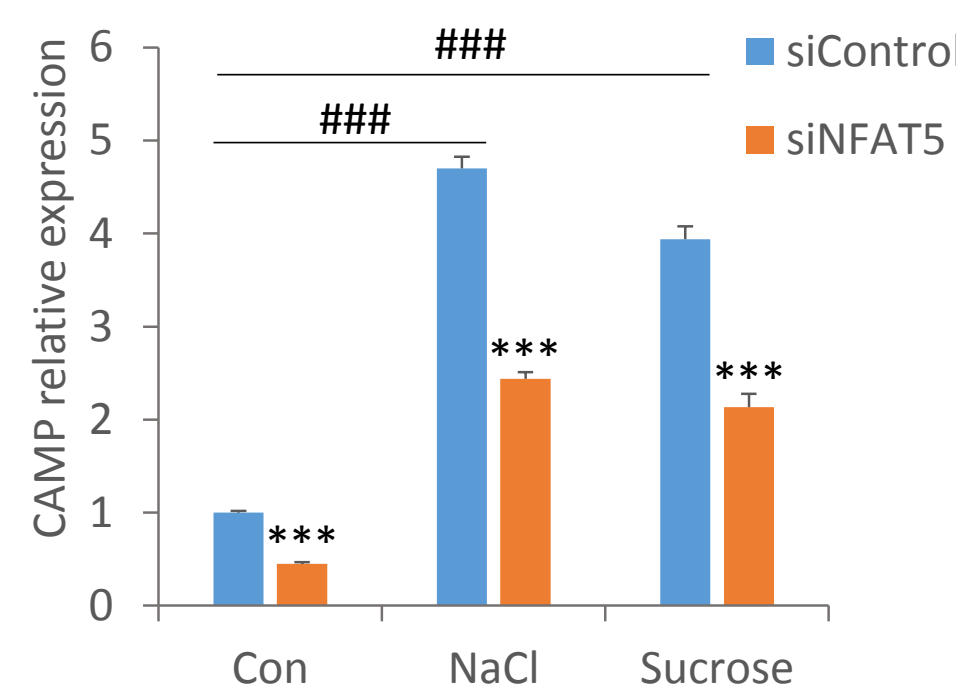

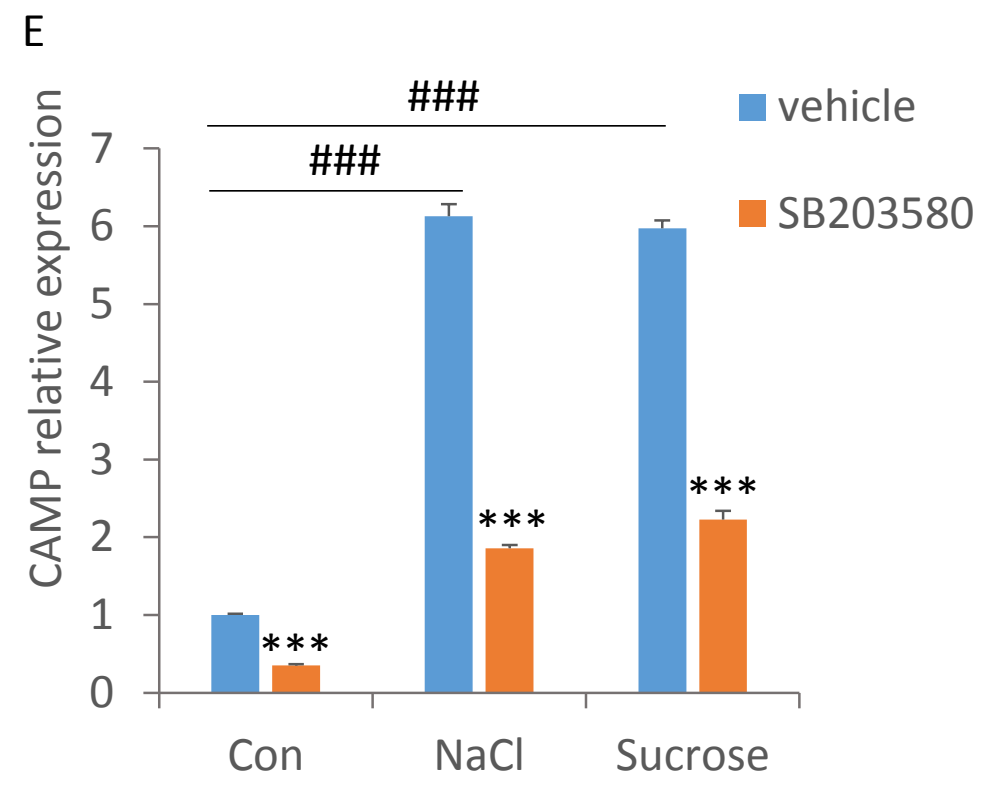

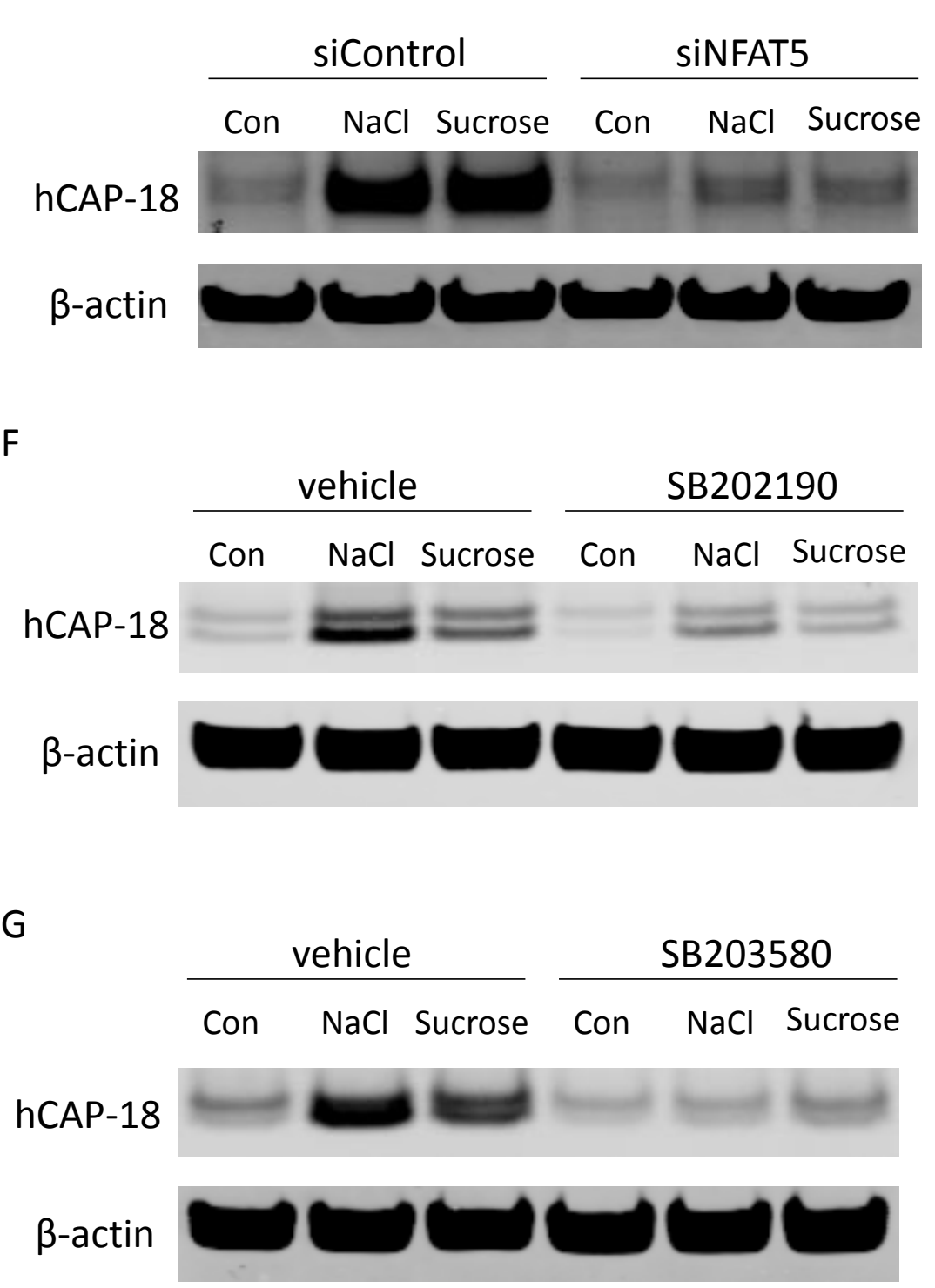

Figure 2 
A

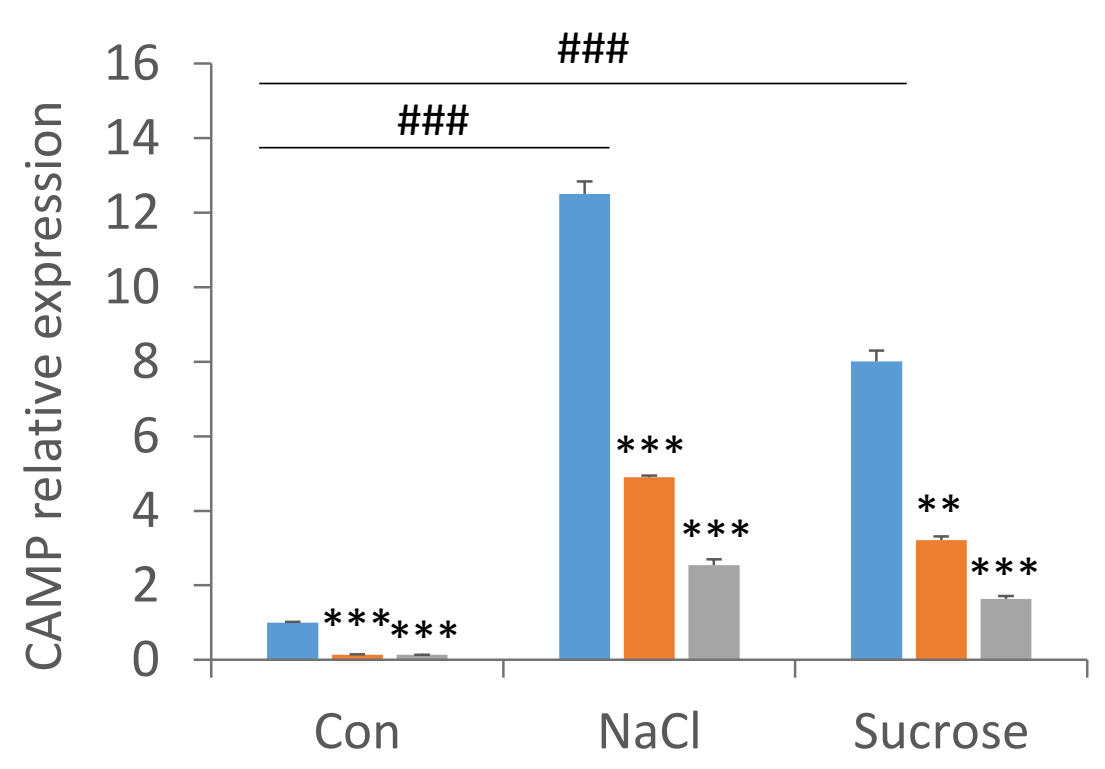

D

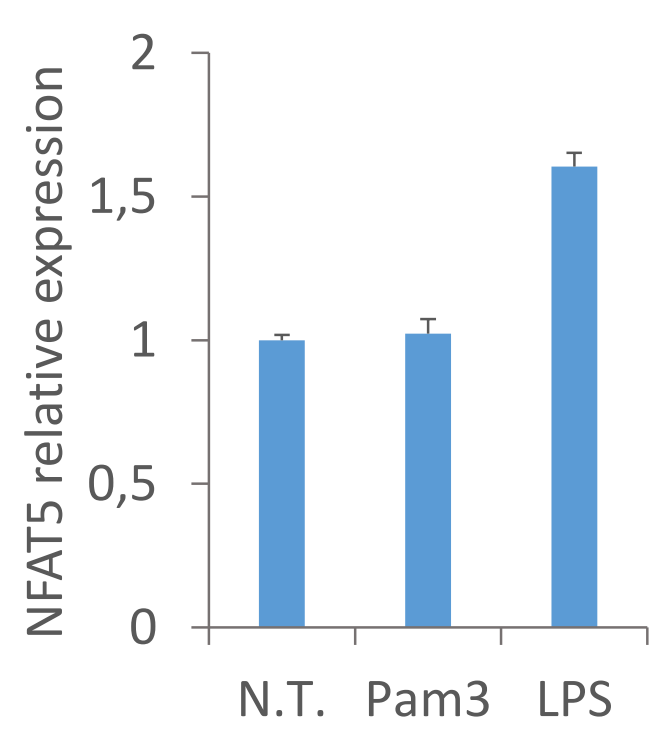

$\square$ N.T.

Dam3

LPS

E

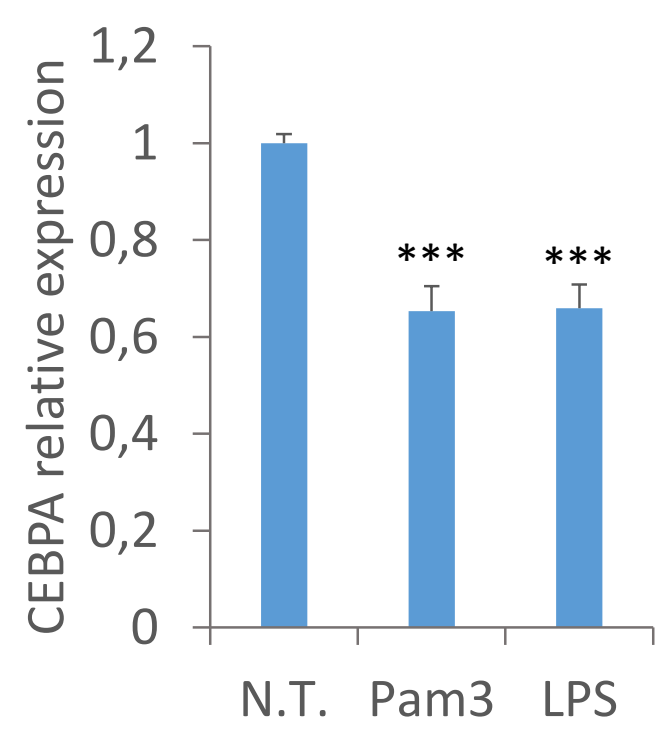

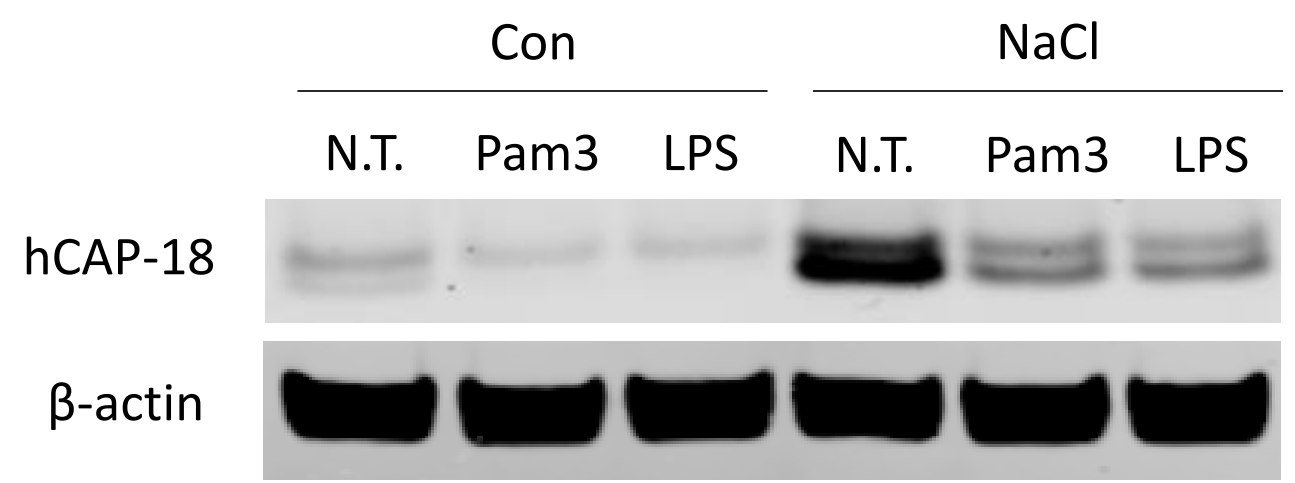

C

\begin{tabular}{|c|c|c|c|c|c|}
\hline \multicolumn{3}{|c|}{ Con } & \multicolumn{3}{|c|}{ Sucrose } \\
\hline N.T. & Pam3 & LPS & N.T. & Pam3 & LPS \\
\hline 5 & & & 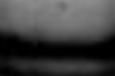 & & \\
\hline
\end{tabular}

Figure 3 

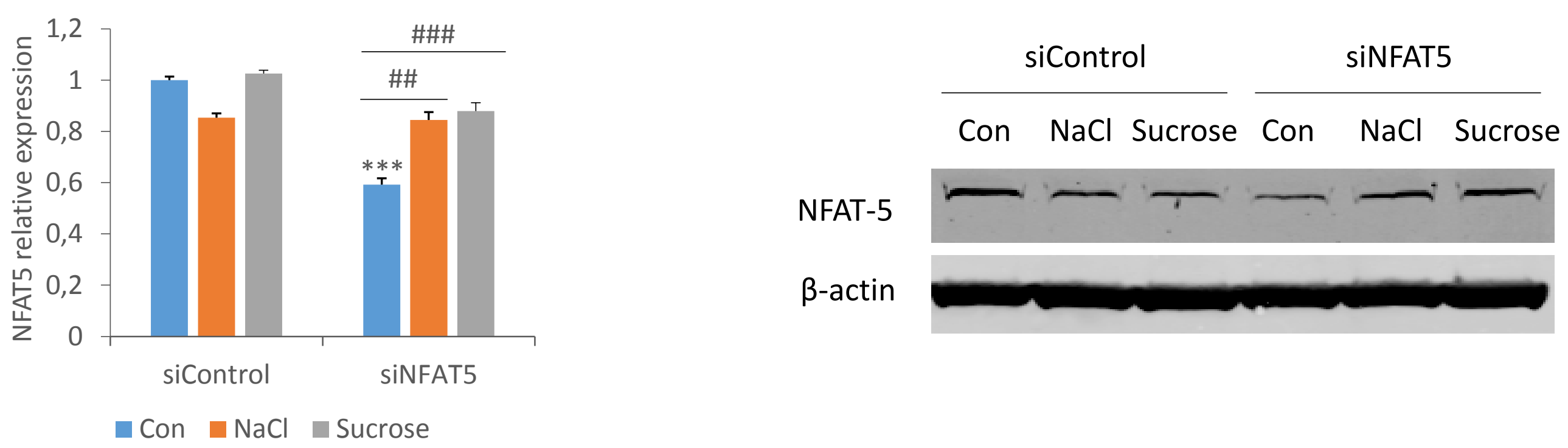

Supplementary Figure 1 